\title{
Poster Exhibit 6: Pediatric Urology
}

Cite as: Can Urol Assoc J 2021;15(6S2):S140-3. http://dx.doi.org/10.5489/cuaj.7405

\begin{abstract}
UP-126
Shared decision-making for pediatric elective penile surgery Wyatt MacNevin' ${ }^{1}$, Amanda MacDonald', Paul Hong', Dawn L. MacLellan ${ }^{3,4}$, Peter A. Anderson ${ }^{3,4}$, Rodrigo Romao ${ }^{3,4}$

${ }^{1}$ Faculty of Medicine, Dalhousie University, Halifax, NS, Canada; ${ }^{2}$ Division of Otolaryngology, Dalhousie University, Halifax, NS, Canada; ${ }^{3}$ Division of Pediatric Urology, IWK Health Centre, Halifax, NS, Canada; ${ }^{4}$ Department of Urology, Dalhousie University, Halifax, NS, Canada

Introduction: Parents act as substitute decision-makers for children undergoing elective surgical treatment, which can lead to decisional conflict (DC). To remedy DC, collaborative shared decision-making (SDM) approaches between physicians and proxy decision-makers may be effective. This study investigates DC and the perception of SDM in elective penile surgery.

Methods: Forty-four parents of children aged $<8$ years undergoing consultation for elective penile surgery at a tertiary pediatric hospital were prospectively enrolled. The SDM process was assessed using SDM-Q-9 (patient) and SDM-Q-Doc (physician) questionnaires and DC was assessed using the SURE screening test. Statistical analysis was performed to determine the relationship between SDM and DC.

Results: Participants included $37(82.2 \%)$ women and $7(15.6 \%)$ men. Participants underwent consultation for circumcision $(n=33,75.0 \%)$ and distal hypospadias repair $(n=11,25.0 \%)$, with $21(47.7 \%)$ participants choosing to proceed with surgery. Seven (15.9\%) participants experienced significant DC. Participant sex and surgery type was not associated with participants experiencing DC $(\mathrm{p}=0.318, \mathrm{p}=0.475)$. The average SDM-Q-9 and SDM-Q-Doc scores were $88.2 \pm 10.0$ and $85.3 \pm 7.4$, respectively, with Spearman correlation analysis showing no relationship between participant and physician perception of SDM involvement $(r=0.168, p=0.276)$. Lower participant (SDM-Q-9: 81.7 $\pm 5.8, \mathrm{p}=0.023$ ) and physician (SDM-Q-Doc: $79.9 \pm 6.9, p=0.029$ ) ratings of SDM were significantly associated with DC. Conclusions: Both parents and pediatric urologists perceived a high degree of SDM pertaining to elective penile surgery in children. In about half the cases, a decision was made to proceed with surgery; only $15 \%$ of parents experienced DC, which was associated with lower participant and physician perceived SDM involvement. Despite high SDM scores overall, discrepancies exist between the perceived physician and participant involvement in the SDM process.
\end{abstract}

\section{UP-127}

Characterizing the safety, effectiveness, and patient population of laparoscopic pyeloplasty for ureteropelvic junction obstruction: A Canadian, single-center, single-surgeon experience Hark Randhawa ${ }^{1}$, Yuding Wang ${ }^{1}$, Melissa McGrath ${ }^{1,2}$, Luis H. Braga ${ }^{1,2,3}$ ${ }^{1}$ Department of Surgery, Urology, McMaster University, Hamilton, ON, Canada; ${ }^{2}$ McMaster Pediatric Surgery Research Collaborative, McMaster University, Hamilton, ON, Canada; ${ }^{3}$ Health, Evidence and Impact, McMaster University, Hamilton, ON, Canada

Introduction: Laparoscopic pyeloplasty (LP)remains an optimal treatment for pediatric patients with ureteropelvic junction obstruction (UPJO). While open and robot-assisted laparoscopic are feasible depending on patient and health systems factors, LP confers decreased morbidity compared to open, and is an important skill for urologists in health systems where access to robotic technology may be limited. We aimed to describe the safety, effectiveness, and clinical characteristics of patients undergoing LP at our tertiary pediatric center.
Methods: We performed a retrospective review of prospectively collected data on patients undergoing LP from 2008-2020 at a single center by one surgeon. Outcomes of interest included operative time (OT), complication rates, and preoperative clinical characteristics, such as symptoms and diagnostic findings. Trends were explored by dividing the patient subset into tertiles and outcomes between groups were compared with Student's t-tests. Results: A total of 154 patients underwent LP with retrograde pyelogram (RPG);109 (71\%) patients were symptomatic preoperatively, the majority of whom either had pain (44\%) or urinary tract infection (UTI) $(19 \%)$. Preoperatively, the mean anteroposterior diameter of the renal pelvis (APD) was $27 \pm 12.1 \mathrm{~mm}$, and among117 patients undergoing renography, mean $\mathrm{t}_{1 / 2}$ was 75 minutes (128). RPG and intraoperative findings revealed that 58 $(38 \%)$ patients had an intrinsic obstruction, $51(33 \%)$ had crossing vessels, and $45(29 \%)$ had UPJO from other etiologies (e.g., polyps). Mean OT was 214 minutes (59) among the whole cohort. There was no statistically significant difference in OT between the first and second tertiles (228 vs. 235 minutes) but there was a difference between the third tertile compared to the prior two $(180$ minutes, $\mathrm{p}<0.001)$. On average, patients were 7.5 years of age at the time of operation; 11 patients were $\leq 1$ year of age, 25 were $1-3$ years, and 118 were $>3$ years. In total, five patients needed a repeat operation, three $(1.9 \%)$ were diagnosed with UTI postoperatively, two $(1.3 \%)$ developed a urinoma, and four $(2.6 \%)$ had omental protrusion at the time of drain removal. The overall complication rate was $9.1 \%$ and the highest number of complications was seen in older patients $(64 \%$ occurred in those $>3$ years).

Conclusions: Low complication and re-operation rates make LP an effective procedure with minimal associated morbidity that can be performed safely in children $<1$ year.

\section{UP-128}

An old friend revisited: The open pyeloplasty in children indications, outcomes, and complications for a large, prospectively collected, single-surgeon experience of 121 patients

Yuding Wang ${ }^{1}$, Roseanne Ferreira ${ }^{7}$, Hark Randhawa ${ }^{7}$, Jaskirat Saini ${ }^{1}$, Melissa Mcgarth', Luis H. Braga?

${ }^{1}$ Department of Surgery, Division of Urology, McMaster University, Hamilton, ON, Canada

Introduction: The open pyeloplasty (OP) had been the gold standard for the management of pediatric ureteropelvic junction obstruction (UPJO). The race for minimally invasive surgery (MIS) saw the OP supplanted in many centers by laparoscopy and robotic-assisted laparoscopy. Studies comparing $\mathrm{OP}$ to emerging MIS techniques have demonstrated no difference in clinical outcomes and complication rates, with a small decrease in length of stay favoring MIS. However, study after study showed patients undergoing MIS were older compared to OP, suggesting a potential selection bias towards delayed intervention. ${ }^{1}$ The following study presents the largest prospective series to date evaluating OP in children.

Methods: We performed a retrospective review of prospectively collected data of consecutive patients undergoing OP between 2008 and 2020 at a single center by a single surgeon. Outcomes of interest included success rate, defined by precent of anteroposterior diameter of the renal pelvis (APD) improvement as a surrogate for obstruction, as well as the rate of complication.

Results: A total of 121 patients underwent OP. Mean age at the time of intervention was 6.2 months, with the majority (62) under six months. Society for Fetal Urology (SFU) grade $3(23 \%)$ and grade $4(76 \%)$ was present at 
the time of surgery, with mean APD of $26.2 \mathrm{~mm}$ (9-70). A MAG3 renal scan was completed in 117 patients (96\%), with the mean renal function of $48 \%$ (19-65). Mean operative time was 139 minutes. Median length of postoperative stay was two days. Most $(93 \%)$ patients showed improved APD by $>40 \%$ by three months postoperatively. Nine patients were found to have non-improving or worsening hydronephrosis, with six patients $(4.9 \%)$ requiring re-operative intervention. Overall, complications rate was $10 \%$, including $4.9 \%$ re-operation rate, $4.1 \%$ related to stent colic. Conclusions: In appropriately selected patients, there remains a role for OP. Low rates of complications and adaptability of technique makes this "old" but tested technique a valuable part of the armamentarium of the modern pediatric urologist.

Reference

1. Mei H, Pu J, Yang C, et al. Laparoscopic vs. open pyeloplasty for ureteropelvic junction obstruction in children: A systematic review and meta-analysis. J Endourol 2011;25:727-36. https://doi. org/10.1089/end.2010.0544

\section{UP-129}

Management of intrinsic vs. extrinsic causes of ureteropelvic junction obstruction in children: Outcomes and complications from a large, prospective, cohort study

Laskirat Saini ${ }^{1}$, Hark Randhawa ${ }^{7}$, Yuding Wang ${ }^{7}$, Melissa Mcgarth ${ }^{7}$, Luis H. Braga

'Department of Surgery, Division of Urology, McMaster University, Hamilton, ON, Canada

Introduction: Ureteropelvic junction obstruction (UPJO) can be caused by intrinsic and or extrinsic (i.e., crossing vessels [CV]) obstruction. Although different in underlying pathology, authors have commonly grouped both etiologies of UPJO together in evaluating treatment options and outcomes. The aim of this study was to evaluate clinical characteristics and outcomes of patients undergoing laparoscopic dismembered pyeloplasty (LDP) for intrinsic and extrinsic UPJO in children.

Methods: We performed a retrospective review of prospectively collected data on patients who underwent LDP between 2008 and 2020 at our institution. The etiology of UPJO was diagnosed by retrograde pyelogram at the beginning of each procedure, and intraoperative findings during laparoscopy. We compared patient characteristics and clinical outcomes between patients with intrinsic vs. extrinsic UPJO using Student's t-tests for continuous variables, and Chi-squared tests for categorical variables. Results: A total of 154 patients underwent LDP during the study period; $103(67 \%)$ were obstructed from an intrinsic cause and $51(33 \%)$ had CV. Patients with an intrinsic cause of UPJO were younger (mean [standard deviation] age 73 [52] months vs. 128 [58] months, $\mathrm{p}<0.001$ ), whereas patients with $\mathrm{CV}$ were more likely to be symptomatic $(92 \%$ vs. $63 \%, \mathrm{p}<0.001)$. Among 117 patients who had a preoperative renal scan, no significant difference was seen in diuretic $\mathrm{t} 1 / 2$ (70 [102] minutes for intrinsic group vs. 87 [173] minutes for $C V, p=0.5$ ). Additionally, both groups had similar rates of Society for Fetal Urology (SFU) grade 4 hydronephrosis on ultrasound preoperatively (44\% intrinsic and $47 \%$ $\mathrm{CV})$. A significant difference was observed in the rate of overall complications ( $7.8 \%$ intrinsic vs. $0 \% \mathrm{CV}, \mathrm{p}=0.041)$; all recurrences $(n=5,3.2 \%)$ occurred in the intrinsic group. No difference in hospital length of stay was observed between groups (median two days).

Conclusions: UPJO secondary to CV is associated with $0 \%$ recurrence compared to $3.2 \%$ for intrinsic obstruction in this large, prospective series. Although distinct entities, both intrinsic obstruction and CV are managed safely and effectively with LDP.

\section{UP-130}

Early complications of non-neonatal circumcisions performed by pediatric urologists: A secondary analysis of the pediatric National Surgical Quality Improvement Program database

Nadeesha Samarasinghe ${ }^{1}$, Jacob Davidson ${ }^{2,3}$, Zhan Tao (Peter) Wang ${ }^{2,4}$, Sumit Dave 2,4

${ }^{1}$ Schulich School of Medicine and Dentistry, Western University, London, ON, Canada; ${ }^{2}$ Department of Surgery, Schulich School of Medicine and Dentistry, Western University, London, ON, Canada; ${ }^{3}$ Division of Pediatric Surgery, Schulich School of Medicine and Dentistry, Western University, London, ON, Canada; ${ }^{4}$ Division of Pediatric Urology, Schulich School of Medicine and Dentistry, Western University, London, ON, Canada Introduction: Male circumcision (MC) is a common surgical procedure in North America. Reported complications of $\mathrm{MC}$ in neonates are relatively low; however, the scant evidence on non-neonatal MC suggests a wide range of complication rates and do not discriminate between surgical provider or technique. This study was designed to estimate early (30-day) complication rates of non-neonatal MC performed by pediatric urologists under general anesthesia (GA).

Methods: A descriptive analysis of secondary data collected from the American College of Surgeons National Surgical Quality Improvement Program ${ }^{\circledast}$ Pediatric (ACS NSQIPP) was performed. ACS NSQIPP datasets are validated and risk-adjusted and in 2017 included 109 institutions worldwide. Patients who underwent elective or medically indicated MC from 2012-2017 were identified using International Classification of Diseases and Related Health Problems and Current Procedures Terminology codes for MC.

Results: A total of $227 \mathrm{MC}$ cases were identified. Median age was 7.2 months (interquartile range $6.2-14.9$ ), the majority $(83.6 \%)$ of patients were not premature at birth, and $81.1 \%$ of patients were Caucasian. When examining 30-day complication rates following $M C$, there was only one case of a superficial surgical site infection. No bleeding complications were reported. Overall, 30-day complication rate was $0.4 \%$.

Conclusions: Short-term complication rates for non-neonatal MC, when performed by pediatric urologists under GA, are lower than that reported in literature. The NSQIPP database make these results generalizable and useful for perioperative counselling of parents seeking elective MC. Furthermore, though delayed complications are not evaluated and controversy continues on adverse impacts of GA, the low complication rate should stimulate discussion on reducing the threshold for performing MC, rather than continuous antibiotic prophylaxis, in pediatric patients with urological conditions predisposed to recurrent urinary tract infections.

\section{UP-131}

\section{International hypospadias practice pattern survey}

Melissa McGrath ${ }^{1,2}$, Ahmed Hadidi' ${ }^{2}$ Christopher Long ${ }^{2}$, Mark Zaontz'2, Tiago Rosito', Antonio Macedo², Grahame Smith², Ibrahim Ulman², Alex Springer ${ }^{2}$, Moneer Hanna2 ${ }^{2}$ Luis H. Braga ${ }^{1,2}$

${ }^{1}$ Surgery, McMaster University, Hamilton, ON, Canada; ${ }^{2}$ Hypospadias International Society, HIS, Frankfurt, Germany

Introduction: A variety of surgical techniques and postoperative care aspects exist for hypospadias (HS) repair. Currently, evidence-based guidelines are lacking, resulting in a diverse clinical practice. We sought to survey members from two international groups, aiming to explore current practice patterns among hypospadiologists.

Methods: An online survey was distributed using REDCapâ to members of the Hypospadias International Society (HIS) and Brazilian School of Pediatric Urology (BSPU) regarding hypospadias repair patterns (pre-, intra-, and postoperatively). To ensure face and content validity, the survey was developed by experts and piloted locally. Anonymized responses were analyzed.

Results: Response rate was 75\% (285/380), with 204 (73\%) respondents working in academic centers. The majority $(169,60 \%)$ performed $<50$ HS repairs/year, with $73 \%$ doing a mean of $16 \pm 21$ proximal cases/year. Caudal blocks are still used by $198(72 \%)$ respondents, while testosterone stimulation is given selectively by $44 \%$. Most $(67 \%)$ would repair glanular $\mathrm{HS}, 52 \%$ using meatal advancement and glanduloplasty (MAGPI), even if parents did not want a circumcision (58\%). Tubularized incised plate 
(TIP) was the technique of choice for distal HS (76\%). Staged repair was the technique of choice for, proximal HS with ventral curvature (VC) $<30^{\circ}(150-36 \%)$ and for those with VC $>30^{\circ}(213-80 \%)$. Most respondents (168-61\%) measured VC by eyeballing. VC $<30^{\circ}$ post-degloving was managed with dorsal plication by $58 \%$ of participants; $58 \%$ and $64 \%$ of respondents divide the urethral plate when the $\mathrm{VC}=30-70^{\circ}$ and for $>70^{\circ}$, respectively. In proximal/scrotal/perineal HS, 152 (54\%) believe the meatus does not need to be brought to tip of the glans. For a TIP repair breakdown, half would do a staged repair. Almost all respondents $(91 \%)$ used postoperative antibiotics and $62 \%$ followed patients past puberty. Conclusions: Over $50 \%$ of respondents agreed in most categories regarding $\mathrm{HS}$ repair, suggesting that multicenter collaboration is more attainable than previously thought. Caudal block, dorsal plication, and testosterone stimulation are still being widely used despite conflicting evidence. Severity of VC dictates procedure choice. Despite hypospadiology being perceived as an art, evidence-based practice is possible, according to this survey's results.

\section{UP-132}

Early postoperative outcomes of ureteric reimplantation surgery for pediatric vesicoureteral reflux: Results from the National Surgical Quality Improvement Program Pediatric database Youshan Ding ${ }^{1}$, Jacob Davidson², Noah Stern ${ }^{3}$, Zhan Tao (Peter) Wang 1,3, Sumit Dave

${ }^{1}$ Faculty of Medicine, Schulich School of Medicine \& Dentistry, Western University, London, ON, Canada; ${ }^{2}$ Division of Pediatric Surgery, Department of Surgery, Schulich School of Medicine \& Dentistry, Western University, London, ON, Canada; ${ }^{3}$ Division of Urology, Department of Surgery, Schulich School of Medicine \& Dentistry, Western University, London, ON, Canada

UP-132. Table 1. Multivariate logistic regression model for risk of unplanned reoperation within 30-day following VUR surgery

\begin{tabular}{lc}
\hline & Relative risk (95\% Cl) \\
\hline Age group & $2.0(1.0-3.8)$ \\
<2 years & $1.1(0.6-2.2)$ \\
2-5 years & $1.0(0.5-2.00)$ \\
6-11 years & Ref. \\
12-18 years & Ref. \\
Sex & $0.8(0.6-1.0)$ \\
Male & \\
Female & $0.6(0.3-1.4)$ \\
Race & $1.5(0.9-2.4)$ \\
Asian and Pacific Islander & Ref. \\
Black or African American & \\
Caucasian & Ref. \\
Surgery type & $0.7(0.4-1.4)$ \\
Open ureteral reimplantation & \\
Laparoscopic/robotic ureteral & \\
reimplantation & Ref. \\
Operative time & $1.8(0.8-4.1)$ \\
Quartile 1 (25th percentile) & $\mathbf{2 . 7 ( 1 . 2 - 6 . 0 )}$ \\
Quartile 2 (25-50th percentile) & $\mathbf{3 . 9}(\mathbf{1 . 7 - 8 . 7 )}$ \\
Quartile 3 (50-75th percentile) & Quartile 4 (75-100th percentile) \\
\hline
\end{tabular}

Increased relative risk indicates an association with unplanned reoperation. Statistically significant confidence intervals $(p<0.05)$ are indicated in bold.
Introduction: Vesicoureteral reflux (VUR) is a common pediatric condition. Surgical options to correct VUR via ureteric reimplantation include open or laparoscopic/robot-assisted ureteric reimplantation (OR/RUR). OR remains the gold standard but use of RUR is increasing. The aim of this study was to use the National Surgical Quality Improvement Program Pediatric (NSQIP-P) database to evaluate early postoperative outcomes of OR vs. RUR.

Methods: A secondary analysis was performed on data from NSQIP-P from 2015-2018. Pediatric patients diagnosed with VUR undergoing surgery were captured using International Classification of Diseases and Current Procedural Terminology codes. Statistical analyses included descriptive statistics, independent samples t-test, and one-way ANOVA for continuous variables and Chi-square tests or Fisher's exact test for categorical variables. Multivariate log regression was used to determine predictors of unplanned reoperation within 30 days.

Results: Overall, 6578 VUR patients were identified. Of these, $95 \%$ received OR and 5\% RUR. Median age at surgery was 4.5 years (interquartile range [IQR] 2.3-7.1); 77\% were female. Compared to OR, children undergoing RUR were older (5.8 vs. 4.5 years, $\mathrm{p}<0.0001$ ), had longer operations ( 162 vs. 139 mins, $p=0.0031$ ), and shorter median hospital stay (LOS) ( 1 vs. 2 days, $\mathrm{p}<0.0001)$. RUR was associated with higher rates of unplanned reoperation ( $4 \%$ vs. $1 \%, p=0.0008)$ and lower rates of urinary tract infection (UTI) ( $1 \%$ vs. $3 \%$. p=0.0097). There were no significant differences in rate of unplanned hospital readmission between OR and RUR ( $5 \%$ vs. 3\%, p=0.1907). On multivariate analysis, younger age and longer operative time were significant predictors of reoperation (Table 1). Conclusions: OR was associated with lower risk of early complications requiring operative intervention. RUR was associated with longer operative time, shorter LOS, and lower UTI rates. Higher risk of early complications needs to be considered when performing RUR in younger children.

\section{UP-133}

Severe ureteropelvic junction obstruction (UPJO)-like hydronephrosis in asymptomatic infants: To operate or not? Abdulrahman Alsabban ${ }^{1,2}$, Todd Dow', Dawn L. MacLellan', Peter A. Anderson', Rodrigo Romao

${ }^{1}$ Urology, Dalhousie University, Halifax, NS, Canada; ${ }^{2}$ Urology, King Abdulaziz University, Jeddah, Saudi Arabia

Introduction: Management of severe hydronephrosis in asymptomatic infants with an impaired drainage pattern on renal scan (ureteropelvic junction obstruction [UPJO]-like) remains controversial. Our goal was to study the natural history of patients with UPJO-like hydronephrosis in a center where a conservative approach with objective indications for pyeloplasty is often used.

Methods: Retrospective chart review of patients undergoing a MAG3 renal scan in a tertiary children's hospital over 16 years. Inclusion criteria were asymptomatic infants with postnatal ultrasound showing Society for Fetal Urology [SFU] grade 3 or 4 unilateral hydronephrosis in the first 18 months of life, with impaired drainage on the renal scan curve. Exclusion criteria were solitary kidney, bilateral cases, presence of ureteric or bladder abnormalities. The primary outcome was pyeloplasty; age at and indication for surgery was also documented. A time-to-event analysis was performed.

Results: A total of 747 patients with renal scan were reviewed; 64 patients met inclusion criteria. Median followup was 40 months. Thirty-eight percent of patients underwent a pyeloplasty at a median age of 21 months. Surgical indications were decreasing renal function $(38 \%)$, febrile urinary tract infections (UTIs) $(20 \%)$, flank pain (12.5\%), and family preference $(4 \%)$. Only $25 \%$ of patients had surgery for worsening hydronephrosis. On time-to-event analysis, the risk of failure increased steadily until 36 months, when it reached $0.42(95 \%$ confidence interval [Cl] $0.30-0.57)$ and plateaued after that. Median differential function on the affected side at last followup was $49.8 \%$ (interquartile range $47.8-52.7 \%$ ) and $49 \%$ patients had improved to SFU grade 2 or less.

Conclusions: Most patients in this series underwent pyeloplasty for an objective indication before three years of age. Half of the patients had a marked reduction in the degree of hydronephrosis, with preservation of 
renal function over time. Our data support a significant role for conservative management with close followup of patients with severe antenatally detected hydronephrosis even in the setting of impaired drainage.

\section{UP-134}

Evaluation of renal measurements of isolated high-grade congenital hydronephrosis $(\mathrm{HGH})$ in both supine and prone positions

Amr Hodhod ${ }^{1}$, Mutaz Farhad ${ }^{1}$, Steven Lu' ${ }^{1}$, Carolina Fermin Risso', Anthony J. Cook', Jarrah Aburezeq ${ }^{1}$, Ravneet Dhaliwal', Bryce A. Weber ${ }^{1}$ ${ }^{1}$ Pediatric Urology, Alberta Children's Hospital, Calgary, AB, Canada Introduction: Some studies have questioned the optimal positioning for the assessment of urinary tract dilatation. The rationale is that the prone position offers better visualization of the kidneys in comparison to the supine position, in which other abdominal organs can interfere with the ultrasound scan. In this study, we evaluated and compared the ultrasound renal measurements in both prone and supine positions.

Methods: We conducted a retrospective review of patients presented with congenital isolated HGH, in the first year of life, from 2017-2019. A control group of patients with normal contralateral kidneys was included. The renal length, anteroposterior diameter of the renal pelvis (APD), parenchymal thickness (PT), and pyramidal thickness (PyT) were measured by a single investigator. APD was measured the renal contour in the mid-renal transverse plane. Both PT and PyT were measured in the midzone of the sagittal plane of the kidney. All measurements were obtained in both supine and prone positions.

Results: Of 81 patients who presented with congenital $\mathrm{HGH}, 65$ patients (117 renal units) were included in our study. Five patients were excluded because the ultrasound was done after the age of one year and 11 excluded because of deficient images. Contralateral normal forty-two renal units were included. Patients' demographics are shown in Table 1. Renal measurements in both positions are presented in Table 2. Prone measurements of renal length, in $\mathrm{HGH}$ and normal groups, were significantly higher (0.003 and 0.005, respectively). In HGH group, although APD measurements in prone position exceeded those in the supine position $(p<0.001)$, PT measurements were significantly greater in the supine position ( $p=0.012$ ). In the normal group, both PT and PyT measurements were lower in a supine position when compared to those in the prone position ( $p=0.006$ and $<0.001$, respectively).

Conclusions: There is a significant difference between renal measurements in both prone and supine positions. However, the clinical impact of this difference should be further evaluated. Due to the prominence of hydronephrosis in the prone position, the PT and PyT measurements were less than the supine ones, which was the inverse in the normal group.

\begin{tabular}{|c|c|c|}
\hline \multicolumn{3}{|c|}{ UP-134. Table 1. Patients' demographics } \\
\hline \multicolumn{2}{|c|}{ Parameter } & Findings \\
\hline \multicolumn{2}{|c|}{ Patients (units) } & 65 patients (117 units*) \\
\hline \multicolumn{2}{|c|}{ Age at ultrasound mean (range) } & 1.24 months $(0.07-8.9)$ \\
\hline \multirow[t]{2}{*}{ Gender } & Male, n (\%) & $50(76.9)$ \\
\hline & Female, n (\%) & $15(23.1)$ \\
\hline \multirow[t]{2}{*}{ Side } & Right, n (\%) & $59(50.4)$ \\
\hline & Left, n (\%) & $58(49.6)$ \\
\hline \multirow[t]{2}{*}{ Laterality } & Bilateral HGH, n (\%) & $10(15.4)$ \\
\hline & Unilateral HGH, n (\%) & $55^{*}(84.6)$ \\
\hline \multirow[t]{3}{*}{ SFU } & SFU 0 (normal), n (\%) & $42(35.9)$ \\
\hline & SFU 3, n (\%) & $25(21.4)$ \\
\hline & SFU 4, n (\%) & $50(42.7)$ \\
\hline
\end{tabular}

\begin{tabular}{|c|c|c|c|}
\hline \multicolumn{4}{|c|}{$\begin{array}{l}\text { UP-134. Table 2. Comparison between renal ultrasound } \\
\text { measurements in both supine and prone positions }\end{array}$} \\
\hline Parameter & $\begin{array}{l}\text { Supine } \\
\text { position }\end{array}$ & $\begin{array}{l}\text { Prone } \\
\text { position }\end{array}$ & $\mathbf{p}$ \\
\hline \multicolumn{4}{|l|}{ HGH group } \\
\hline $\begin{array}{l}\text { Renal length } \\
\text { mean(range) }\end{array}$ & $\begin{array}{l}68.22 \mathrm{~mm} \\
(46.1-100.4)\end{array}$ & $\begin{array}{l}69.52 \mathrm{~mm} \\
(48-102)\end{array}$ & 0.003 \\
\hline APD mean (range) & $\begin{array}{c}20.8 \mathrm{~mm} \\
(7-63)\end{array}$ & $\begin{array}{l}22.95 \mathrm{~mm} \\
(9.7-66)\end{array}$ & $<0.001$ \\
\hline $\begin{array}{l}\text { Parenchymal thickness } \\
\text { mean (range) }\end{array}$ & $\begin{array}{l}6.2 \mathrm{~mm} \\
(2.8-10)\end{array}$ & $\begin{array}{c}5.8 \mathrm{~mm} \\
(2.2-11 \mathrm{~mm})\end{array}$ & 0.012 \\
\hline $\begin{array}{l}\text { Pyramidal thickness } \\
\text { mean (range) }\end{array}$ & $1.9 \mathrm{~mm}(0-4.9)$ & $1.8 \mathrm{~mm}(0-5)$ & 0.9 \\
\hline \multicolumn{4}{|l|}{ Normal group } \\
\hline $\begin{array}{l}\text { Renal length mean } \\
\text { (range) }\end{array}$ & $\begin{array}{l}54.96 \mathrm{~mm} \\
(36.6-69.5)\end{array}$ & $\begin{array}{c}56.3 \mathrm{~mm} \\
(37-74)\end{array}$ & 0.005 \\
\hline APD mean (range) & $2.1 \mathrm{~mm}(0-7)$ & $2.2 \mathrm{~mm}(0-8)$ & 0.28 \\
\hline $\begin{array}{l}\text { Parenchymal thickness } \\
\text { mean (range) }\end{array}$ & $\begin{array}{c}9.9 \mathrm{~mm} \\
(6-13)\end{array}$ & $\begin{array}{l}10.6 \mathrm{~mm} \\
(6.6-13)\end{array}$ & 0.006 \\
\hline $\begin{array}{l}\text { Pyramidal thickness } \\
\text { mean (range) }\end{array}$ & $5.9 \mathrm{~mm}(4-9)$ & $\begin{array}{c}6.6 \mathrm{~mm} \\
(3.8-10.1)\end{array}$ & $<0.001$ \\
\hline
\end{tabular}

